\title{
Non-meridional epimorphisms of knot groups
}

\author{
JAE CHOON CHA \\ MASAAKI SUZUKI
}

\begin{abstract}
In the study of knot group epimorphisms, the existence of an epimorphism between two given knot groups is mostly (if not always) shown by giving an epimorphism which preserves meridians. A natural question arises: is there an epimorphism preserving meridians whenever a knot group is a homomorphic image of another? We answer in the negative by presenting infinitely many pairs of prime knot groups $\left(G, G^{\prime}\right)$ such that $G^{\prime}$ is a homomorphic image of $G$ but no epimorphism of $G$ onto $G^{\prime}$ preserves meridians.
\end{abstract}

20F34, 20J05, 57M05, 57M25

\section{Introduction}

For a knot $K$ in $S^{3}$, its knot group $G(K)$ is defined by $G(K)=\pi_{1}\left(S^{3}-K\right)$. The study of knot groups has a long history, from the beginning of modern knot theory. In particular, recently, epimorphisms of knot groups have been receiving much attention. A key problem is to determine when there is an epimorphism between two knot groups. For prime knots, which are of the most interest, it is well known that a partial order $\geq$ is obtained by defining $K \geq K^{\prime}$ if there is an epimorphism $G(K) \rightarrow G\left(K^{\prime}\right)$ (see, for instance, Ohtsuki, Riley and Sakuma [18, page 422]).

There is a fair amount of recent work on this in the literature. In their remarkable work [1], Agol and Liu proved a long-standing conjecture of Simon that a knot group surjects onto only finitely many knot groups. It follows that for any prime knot $K$, there are only finitely many prime knots less than or equal to $K$. Together with Kitano, Horie, Matsumoto and Wada, the second author investigated pairs of prime knots with 11 crossings or less whose knot groups admit epimorphisms [14; 9; 16]. In particular, they constructed many explicit examples of epimorphisms between knot groups. Gonzaléz-Acuña and Ramírez studied which knot groups (particularly those of 2-bridge knots) admit epimorphisms onto torus knot groups [7; 8]. In work of Ohtsuki, Riley and Sakuma [18], Hoste and Shanahan [11], and Lee and Sakuma [17], systematic constructions of epimorphisms between 2-bridge knot (and link) groups were presented and studied. Silver and Whitten [20;21] studied knot group epimorphisms preserving 
peripheral structure; in particular, they showed that such epimorphisms give rise to a partial order on the set of all knots.

\section{Meridional epimorphisms}

Interestingly, most (if not all) results in the literature stating that a knot is less than or equal to another are shown by presenting an epimorphism which preserves meridians. To be more precise, we use the following terms: we call an element $[\alpha] \in G(K)$ a meridian if $\alpha$ is freely homotopic to a meridian curve lying on the boundary of a tubular neighborhood of $K$, and we say that a homomorphism $G(K) \rightarrow G\left(K^{\prime}\right)$ is meridional if a meridian in $G(K)$ is sent to a meridian in $G\left(K^{\prime}\right)$. In this paper knots are unoriented, so that a meridian may be endowed with any orientation.

The following natural question arises:

Question Is there a meridional epimorphism $G(K) \rightarrow G\left(K^{\prime}\right)$ whenever there is an epimorphism $G(K) \rightarrow G\left(K^{\prime}\right)$ ?

We remark that it does not ask whether all knot group epimorphisms are meridional; it is known that there exist non-meridional epimorphisms of knot groups. For instance, see work of Johnson and Livingston [13]. We also remark that meridional epimorphisms can be related to geometric properties, for example, periods of knots and degree-one maps between knot exteriors. See Kitano and Suzuki [15] for details.

There are several results supporting an affirmative answer to the above question. For any previously known example of a knot group $G(K)$ which admits an epimorphism onto $G\left(K^{\prime}\right)$, there exists a meridional epimorphism of $G(K)$ onto $G\left(K^{\prime}\right)$. In particular, all the epimorphisms found in $[9 ; 14 ; 16]$ for groups of prime knots with 11 or less crossings are meridional. For torus knot groups, there is a meridional epimorphism whenever there is an epimorphism [21]. Also, the knot group epimorphisms in [7; 8; $17 ; 18]$ are all meridional. An epimorphism between nontrivial knot groups preserving peripheral structure in the sense of $[20 ; 21]$ is known to be meridional [20, proof of Theorem 4.1; 11, Theorem 2.1]. We also remark that epimorphisms preserving peripheral structure, particularly meridional epimorphisms of prime knot groups, can be studied via maps of 3-manifolds with well-defined degree.

Our main result is, nevertheless, that the answer is in the negative.

Theorem 1.1 There are infinitely many distinct pairs of prime knots $\left(K, K^{\prime}\right)$ for which there is an epimorphism of $G(K)$ onto $G\left(K^{\prime}\right)$ but there is no meridional epimorphism of $G(K)$ onto $G\left(K^{\prime}\right)$. 
The proof of Theorem 1.1 proceeds as follows. In Section 2, we give constructions of pairs of knot groups which admit non-meridional epimorphisms. In Section 3, we detect the non-existence of meridional epimorphisms using twisted Alexander polynomials. This method allows us to obtain finitely many (in fact two) "seed" examples. We remark that it depends on heavy computation infeasible by hand, and hence it seems unable to detect infinitely many cases in this way. In Section 4, we present a geometric method to produce, from the seed examples, infinitely many pairs of knot groups which admit non-meridional epimorphisms but do not admit meridional epimorphisms. In the appendix we present computational results of certain twisted Alexander polynomials which are used to prove the non-existence of a meridional epimorphism.

Acknowledgements Cha was partially supported by NRF grants 2013067043 and 2013053914. Suzuki was partially supported by KAKENHI (number 24740035), Japan Society for the Promotion of Science, Japan.

\section{Construction of non-meridional knot group epimorphisms}

In this section we give certain explicit examples non-meridional epimorphisms of knot groups, for some of which we will show the non-existence of meridional epimorphisms in the next section.

\subsection{First example on the trefoil knot}

In this subsection we describe the first successful example of a pair of knots satisfying Theorem 1.1, which we found by ad-hoc trial and error attempts aided by a computer.

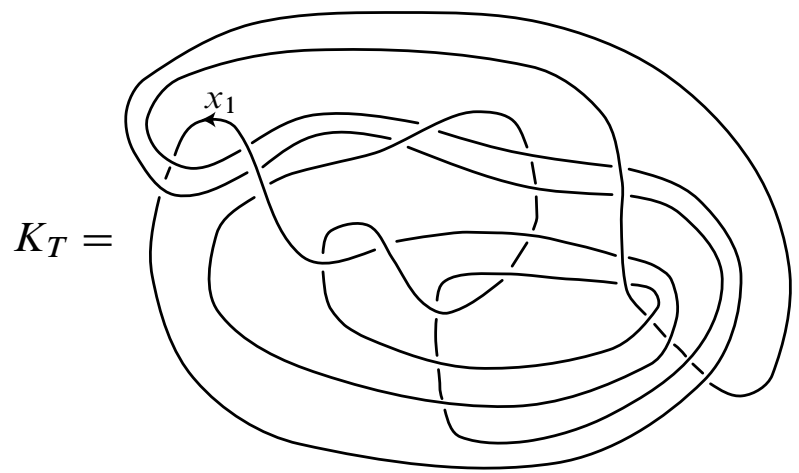

Figure 1: A knot $K_{T}$. 
Consider the knot $K_{T}$ shown in Figure 1. The Wirtinger presentation of the knot group $G\left(K_{T}\right)$ obtained from Figure 1 has 24 generators $x_{1}, x_{2}, \ldots, x_{24}$, and 24 relators

$\begin{array}{llll}x_{6} x_{2} \bar{x}_{6} \bar{x}_{1}, & x_{10} x_{2} \bar{x}_{10} \bar{x}_{3}, & x_{6} x_{3} \bar{x}_{6} \bar{x}_{4}, & x_{22} x_{4} \bar{x}_{22} \bar{x}_{5}, \\ x_{1} x_{6} \bar{x}_{1} \bar{x}_{5}, & x_{17} x_{7} \bar{x}_{17} \bar{x}_{6}, & x_{23} x_{7} \bar{x}_{23} \bar{x}_{8}, & x_{13} x_{9} \bar{x}_{13} \bar{x}_{8}, \\ x_{3} x_{9} \bar{x}_{3} \bar{x}_{10}, & x_{1} x_{10} \bar{x}_{1} \bar{x}_{11}, & x_{22} x_{12} \bar{x}_{22} \bar{x}_{11}, & x_{6} x_{13} \bar{x}_{6} \bar{x}_{12}, \\ x_{23} x_{14} \bar{x}_{23} \bar{x}_{13}, & x_{17} x_{14} \bar{x}_{17} \bar{x}_{15}, & x_{18} x_{16} \bar{x}_{18} \bar{x}_{15}, & x_{6} x_{17} \bar{x}_{6} \bar{x}_{16}, \\ x_{1} x_{17} \bar{x}_{1} \bar{x}_{18}, & x_{16} x_{19} \bar{x}_{16} \bar{x}_{18}, & x_{24} x_{19} \bar{x}_{24} \bar{x}_{20}, & x_{12} x_{21} \bar{x}_{12} \bar{x}_{20}, \\ x_{4} x_{21} \bar{x}_{4} \bar{x}_{22}, & x_{1} x_{23} \bar{x}_{1} \bar{x}_{22}, & x_{6} x_{23} \bar{x}_{6} \bar{x}_{24}, & x_{18} x_{24} \bar{x}_{18} \bar{x}_{1} .\end{array}$

Here the generators are ordered along the orientation, starting from the generator $x_{1}$ shown in Figure 1. In the relators $\bar{x}$ denotes the inverse of $x$.

Recall that the trefoil knot $T$ has the following Wirtinger presentation:

$$
G(T)=\left\langle y_{1}, y_{2} \mid y_{1} y_{2} y_{1}=y_{2} y_{1} y_{2}\right\rangle .
$$

We define a map $f: G\left(K_{T}\right) \rightarrow G(T)$ as follows:

$$
\begin{aligned}
& f\left(x_{1}\right)=y_{1} y_{2} \bar{y}_{1} y_{2} \bar{y}_{1}, \\
& f\left(x_{13}\right)=y_{1} y_{2} \bar{y}_{1} y_{2} \bar{y}_{1}, \\
& f\left(x_{2}\right)=y_{1} \bar{y}_{2} y_{1} \bar{y}_{2} y_{1} \bar{y}_{2} \bar{y}_{1} y_{2}^{3} \\
& f\left(x_{14}\right)=y_{1} \bar{y}_{2} y_{1} \bar{y}_{2}^{3} y_{1} y_{2} \bar{y}_{1} y_{2} \bar{y}_{1} y_{2}^{3} \bar{y}_{1} y_{2} \bar{y}_{1}, \\
& \cdot \bar{y}_{1} y_{2} \bar{y}_{1} y_{2} \bar{y}_{1}, \\
& f\left(x_{15}\right)=y_{1} y_{2} \bar{y}_{1} y_{2} \bar{y}_{1} \text {, } \\
& f\left(x_{3}\right)=y_{1} y_{2} \bar{y}_{1} y_{2} \bar{y}_{1} \text {, } \\
& f\left(x_{16}\right)=y_{1} \bar{y}_{2}^{2} y_{1} y_{2} \bar{y}_{1} y_{2} \bar{y}_{1} y_{2}^{2} \bar{y}_{1} \text {, } \\
& f\left(x_{4}\right)=y_{1} \bar{y}_{2} y_{1} y_{2} \bar{y}_{1} y_{2} \bar{y}_{1} y_{2} y_{1} \bar{y}_{2} \bar{y}_{1} y_{2} \bar{y}_{1}, \quad f\left(x_{17}\right)=y_{1} \bar{y}_{2} y_{1} \bar{y}_{2} y_{1} \bar{y}_{2} \bar{y}_{1} \bar{y}_{2} y_{1} y_{2} \bar{y}_{1} \\
& f\left(x_{5}\right)=y_{2} y_{1} y_{2} \bar{y}_{1} y_{2} \bar{y}_{1} y_{2} y_{1} \bar{y}_{2} \bar{y}_{1} \bar{y}_{2} \text {, } \\
& \text { - } y_{2} \bar{y}_{1} y_{2} y_{1} y_{2} \bar{y}_{1} y_{2} \bar{y}_{1} y_{2} \bar{y}_{1} \text {, } \\
& f\left(x_{6}\right)=y_{1} \bar{y}_{2} y_{1} y_{2} \bar{y}_{1} y_{2} \bar{y}_{1} y_{2} \bar{y}_{1} \text {, } \\
& f\left(x_{18}\right)=y_{2}^{2} \bar{y}_{1} \text {, } \\
& f\left(x_{7}\right)=y_{1} \bar{y}_{2} y_{1} \bar{y}_{2}^{3} y_{1} y_{2}^{2} \bar{y}_{1} \bar{y}_{1} y_{2}^{3} \bar{y}_{1} y_{2} \bar{y}_{1} \text {, } \\
& f\left(x_{19}\right)=y_{1} \bar{y}_{2}^{2} y_{1} \bar{y}_{2} y_{1} \bar{y}_{2} \bar{y}_{1} y_{2}^{3} \bar{y}_{1} y_{2} \bar{y}_{1} y_{2}^{2} \bar{y}_{1} \text {, } \\
& f\left(x_{8}\right)=y_{1} \bar{y}_{2} y_{1} y_{2} \bar{y}_{1} y_{2} \bar{y}_{1} y_{2} \bar{y}_{1} \text {, } \\
& f\left(x_{20}\right)=y_{2}^{2} \bar{y}_{1} \text {, } \\
& f\left(x_{9}\right)=y_{1} \bar{y}_{2} y_{1} \bar{y}_{2}^{2} y_{1} y_{2} \bar{y}_{1} y_{2} \bar{y}_{1} \\
& f\left(x_{21}\right)=y_{1} \bar{y}_{2} y_{1} y_{2} \bar{y}_{1} \bar{y}_{2} y_{1} \bar{y}_{2} y_{1} \bar{y}_{2} \bar{y}_{1} y_{2} \bar{y}_{1} \\
& \text { - } y_{2}^{2} \bar{y}_{1} y_{2} \bar{y}_{1} \text {, } \\
& \text { - } y_{2} y_{1} y_{2} \bar{y}_{1} y_{2} \bar{y}_{1} y_{2} y_{1} \bar{y}_{2} \bar{y}_{1} y_{2} \bar{y}_{1} \text {, } \\
& f\left(x_{10}\right)=y_{1} \bar{y}_{2} y_{1} y_{2} \bar{y}_{1} y_{2} \bar{y}_{1} y_{2} \bar{y}_{1}, \\
& f\left(x_{22}\right)=y_{2}^{2} \bar{y}_{1}, \\
& f\left(x_{11}\right)=y_{1} y_{2} y_{2} \bar{y}_{1}^{2} \text {, } \\
& f\left(x_{23}\right)=y_{1} \bar{y}_{2} y_{1} \bar{y}_{2} \bar{y}_{1} y_{2}^{3} \bar{y}_{1} y_{2} \bar{y}_{1}, \\
& f\left(x_{12}\right)=y_{1} \bar{y}_{2}^{2} y_{1} y_{2}^{2} \bar{y}_{1}^{2} y_{2}^{2} \bar{y}_{1} \text {, } \\
& f\left(x_{24}\right)=y_{1} \bar{y}_{2} y_{1} y_{2} \bar{y}_{1} \bar{y}_{1} y_{2}^{2} y_{1} \bar{y}_{2} \bar{y}_{1} y_{2} \bar{y}_{1} \text {. }
\end{aligned}
$$

Theorem 2.1 The map $f: G\left(K_{T}\right) \rightarrow G(T)$ is a non-meridional epimorphism. 
Proof It is shown that $f$ is a group homomorphism by directly verifying that the relators of $G\left(K_{T}\right)$ vanish under $f$. For instance, we have

$$
\begin{array}{r}
f\left(x_{6} x_{2} \bar{x}_{6} \bar{x}_{1}\right)=y_{1} \bar{y}_{2} y_{1} y_{1} \bar{y}_{1} y_{1} \bar{y}_{1} y_{1} \bar{y}_{1} \cdot y_{1} \bar{y}_{2} y_{1} \bar{y}_{2} y_{1} \bar{y}_{2} \bar{y}_{1} y_{1}^{3} \bar{y}_{1} y_{1} \bar{y}_{1} y_{1} \bar{y}_{1} \\
\cdot y_{1} \bar{y}_{2} y_{1} \bar{y}_{2} y_{1} \bar{y}_{2} \bar{y}_{1} y_{1} \bar{y}_{1} \cdot y_{1} \bar{y}_{2} y_{1} \bar{y}_{2} \bar{y}_{1}=e
\end{array}
$$

and

$$
\begin{array}{r}
f\left(x_{10} x_{2} \bar{x}_{10} \bar{x}_{3}\right)=y_{1} \bar{y}_{2} y_{1} y_{2} \bar{y}_{1} y_{2} \bar{y}_{1} y_{2} \bar{y}_{1} \cdot y_{1} \bar{y}_{2} y_{1} \bar{y}_{2} y_{1} \bar{y}_{2} \bar{y}_{1} y_{2}^{3} \bar{y}_{1} y_{2} \bar{y}_{1} y_{2} \bar{y}_{1} \\
\cdot y_{1} \bar{y}_{2} y_{1} \bar{y}_{2} y_{1} \bar{y}_{2} \bar{y}_{1} y_{2} \bar{y}_{1} \cdot y_{1} \bar{y}_{2} y_{1} \bar{y}_{2} \bar{y}_{1}=e,
\end{array}
$$

and so forth.

To show that $f$ is an epimorphism, we explicitly describe elements of $G\left(K_{T}\right)$ which are sent to generators of $G(T)$ :

$$
\begin{aligned}
f\left(x_{18} x_{6} \bar{x}_{1} \bar{x}_{1} x_{18} x_{6} \bar{x}_{1}\right)= & y_{2}^{2} \bar{y}_{1} \cdot y_{1} \bar{y}_{2} y_{1} y_{2} \bar{y}_{1} y_{2} \bar{y}_{1} y_{2} \bar{y}_{1} \cdot y_{1} \bar{y}_{2} y_{1} \bar{y}_{2} \bar{y}_{1} \cdot y_{1} \bar{y}_{2} y_{1} \bar{y}_{2} \bar{y}_{1} \\
& \cdot y_{2}^{2} \bar{y}_{1} \cdot y_{1} \bar{y}_{2} y_{1} y_{2} \bar{y}_{1} y_{2} \bar{y}_{1} \bar{y}_{1} \cdot y_{1} \bar{y}_{2} y_{1} \bar{y}_{2} \bar{y}_{1} \\
= & y_{2} y_{1} y_{2} \bar{y}_{1} \bar{y}_{2} y_{1} \bar{y}_{2} \bar{y}_{1} y_{2} y_{1} y_{2} \bar{y}_{1} \bar{y}_{1} \\
= & y_{1} y_{2} y_{1} \bar{y}_{1} \bar{y}_{2} y_{1} \bar{y}_{2} \bar{y}_{1} y_{1} y_{2} y_{1} \bar{y}_{1} \bar{y}_{1}=y_{1} .
\end{aligned}
$$

Similarly we have

$$
f\left(x_{1} \bar{x}_{6} \bar{x}_{18} x_{1} x_{18} x_{6} \bar{x}_{1}^{2} x_{18} x_{6} \bar{x}_{1}\right)=y_{2} .
$$

Although we will show that there is no meridional epimorphism of $G\left(K_{T}\right)$ onto $G(T)$ in the next section, we present here a simple direct proof that our $f$ is not meridional. Define a representation $\rho: G(T) \rightarrow \operatorname{SL}(2, \mathbb{Z})$ by

$$
\rho\left(y_{1}\right)=\left[\begin{array}{ll}
1 & 1 \\
0 & 1
\end{array}\right], \quad \rho\left(y_{2}\right)=\left[\begin{array}{cc}
1 & 0 \\
-1 & 1
\end{array}\right] .
$$

It is straightforward to verify that $\rho$ is well-defined. The image of $f\left(x_{1}\right)$ under $\rho$ is given by

$$
\rho\left(f\left(x_{1}\right)\right)=\rho\left(y_{1} y_{2} \bar{y}_{1} y_{2} \bar{y}_{2}\right)=\left[\begin{array}{ll}
-1 & 2 \\
-3 & 5
\end{array}\right] .
$$

It has trace 4, while the trace of $\rho\left(y_{1}\right)$ is 2 . It follows that the image $f\left(x_{1}\right)$ of the meridian $x_{1}$ of $K_{T}$ is not conjugate to the meridian $y_{1}$ of $T$.

We remark that $K_{T}$ is a hyperbolic knot, according to SnapPy [4], and consequently $K_{T}$ is prime. 


\subsection{Construction using normal generators and Johnson's method}

To describe the second successful example satisfying Theorem 1.1, we employ a more systematic construction which combines algebraic computations in knot groups and geometric realization arguments. In the first step we construct a non-meridional normal generator, and in the second step we construct knot group homomorphisms realizing the normal generator as the image of a meridian.

Finding pseudo-meridians: twist knots It is well known that a meridian is a normal generator of a knot group. We call a normal generator of a knot group a pseudomeridian, that is, $w \in G(K)$ is called a pseudo-meridian if $G(K) /\langle w\rangle$ is trivial, where $\langle w\rangle$ is the normal closure of $w$.

We will present useful pseudo-meridians of twist knots. Let $J(2,2 q)$ be the twist knot shown in Figure $2(q \in \mathbb{Z})$. For example, $J(2,0)$ is the trivial knot, $J(2,2)$ is the trefoil knot, and $J(2,-2)$ is the figure-eight knot. The presentation of $G(J(2,2 q))$ is given by

$$
G(J(2,2 q))=\left\langle a, b \mid w^{q} a=b w^{q}\right\rangle, \quad w=\left[b, a^{-1}\right] .
$$

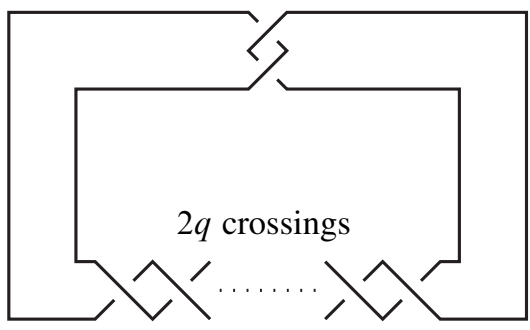

Figure 2: The twist knot $J(2,2 q)$.

\section{Proposition 2.2 Let}

$$
g_{1}= \begin{cases}w^{q} a & \text { if } q>0 \\ \bar{w}^{q} b & \text { if } g<0 .\end{cases}
$$

Then $g_{1}$ is a pseudo-meridian, but not a meridian for $q \neq 0$.

Proof First we show that $g_{1}$ is a pseudo-meridian by verifying that $G(J(2,2 q)) /\left\langle g_{1}\right\rangle$ is trivial. In the quotient, $g_{1}=e$, that is, $w^{q}=\bar{a}$ if $q>0$. Then the relation of the twist knot group gives $\bar{a} a=b \bar{a}$. This implies that $b=a$ and $w=[b, \bar{a}]=e$. Therefore $a=b=\bar{w}^{q}=e$. Hence $g_{1}$ is a pseudo-meridian. Similarly, we can show that $g_{1}=\bar{w}^{q} b$ is a pseudo-meridian for $q<0$. 
Next we show that $g_{1}$ is not conjugate to $a$, that is, $g_{1}$ is not a meridian of the twist knot. Let $\rho: G(J(2,2 q)) \rightarrow \operatorname{SL}(2, \mathbb{C})$ be defined by

$$
\rho(a)=\left[\begin{array}{ll}
1 & 1 \\
0 & 1
\end{array}\right], \quad \rho(b)=\left[\begin{array}{cc}
1 & 0 \\
-u & 1
\end{array}\right], \quad u \in \mathbb{C}-\{0\} .
$$

We set $\phi_{q}(u)$ to be the $(1,1)$-entry of $\rho\left(w^{q}\right)$. It is easy to see that if $u=0$, then $\rho$ is not a representation. More precisely, Riley [19] showed that $\rho$ is a non-abelian parabolic representation if and only if $\phi_{q}(u)=0$. Moreover, Hoste and Shanahan [10] proved that $\phi_{q}(u)$ is irreducible and that $\operatorname{deg} \phi_{q}(u)$ is $2 q-1$ if $q>0$ and $2|q|$ if $q<0$.

We define a polynomial $p_{q}(u)$ by

$$
p_{q}(u)= \begin{cases}\operatorname{tr}\left(\rho\left(w^{q} a\right)\right)-\operatorname{tr}(\rho(a))=\operatorname{tr}\left(\rho\left(w^{q} a\right)\right)-2 & \text { if } q>0, \\ \operatorname{tr}\left(\rho\left(\bar{w}^{q} b\right)\right)-\operatorname{tr}(\rho(a))=\operatorname{tr}\left(\rho\left(\bar{w}^{q} b\right)\right)-2 & \text { if } q<0 .\end{cases}
$$

First, consider the case $q>0$. The Cayley-Hamilton theorem gives us

$$
\rho(w)^{2}-(\operatorname{tr} \rho(w)) \rho(w)+I=0 .
$$

The trace of $\rho(w)$ is $u^{2}+2$. Multiplying both sides by $\rho\left(w^{q-2} a\right)$, we obtain

$$
\rho\left(w^{q} a\right)=\left(u^{2}+2\right) \rho\left(w^{q-1} a\right)-\rho\left(w^{q-2} a\right) .
$$

Taking the trace of the both sides and using the definition of $p_{q}(u)$, we obtain a recursion formula for $p_{q}(u)$ :

$$
p_{q}(u)=\left(u^{2}+2\right) p_{q-1}(u)-p_{q-2}(u)+2 u^{2}
$$

(compare [10]). Since $p_{1}(u)=2 u^{2}$ and $p_{2}(u)=2 u^{4}+6 u^{2}$, we conclude that $p_{q}(u)$ has a factor $u^{2}$ and $\operatorname{deg} p_{q}(u)$ is $2 q$. Then $p_{q}(u)$ can be written as

$$
p_{q}(u)=u^{2} \bar{p}_{q}(u),
$$

and the degree of $\bar{p}_{q}(u)$ is $2 q-2$, which is less than $\operatorname{deg} \phi_{q}(u)$. Since $\phi_{q}(u)$ is irreducible, $\phi_{q}(u)$ does not have a multiple root, that is, $\phi_{q}(u)$ has $2 q-1$ distinct roots, which are not zero. Hence there exists at least one root $u \in \mathbb{C}$ of $\phi_{q}(u)=0$ such that $p_{q}(u) \neq 0$, namely,

$$
\operatorname{tr}\left(\rho\left(w^{q} a\right)\right) \neq \operatorname{tr}(\rho(a)) .
$$

This implies that $w^{q} a$ is not conjugate to $a$. Similarly, we prove the statement for the case $q<0$. The recursion formula of $p_{q}(u)$ is given by

$$
p_{q}(u)=\left(u^{2}+2\right) p_{q+1}(u)-p_{q+2}(u)+2 u^{2} .
$$


By straightforward computation, $p_{-1}(u)=0$ and $p_{-2}(u)=2 u^{2}$. Then the same argument as the case $q>0$ holds. Therefore $\bar{w}^{q} b$ is not conjugate to $a$. This completes the proof.

We can produce a generating set of the twist knot group by conjugating $g_{1}$. In the case $q>0$, the relation can be written as $\left(g_{1} \bar{a}\right) a=b\left(g_{1} \bar{a}\right)$. Then $b=g_{1} a \bar{g}_{1}$ and

$$
w=[b, \bar{a}]=g_{1} a \bar{g}_{1} \cdot \bar{a} \cdot g_{1} \bar{a} \bar{g}_{1} \cdot a=\left[g_{1}, a\right]\left[g_{1}, \bar{a}\right] .
$$

Therefore we obtain

$$
\begin{aligned}
a & =\left(\left[\bar{a}, g_{1}\right]\left[a, g_{1}\right]\right)^{q} g \\
& =\bar{a} g_{1} a \cdot \bar{g}_{1} \cdot a g_{1} \bar{a} \cdot \bar{g}_{1} \ldots \bar{a} g_{1} a \cdot \bar{g}_{1} \cdot a g_{1} \bar{a} \\
& =\left(g_{2} \bar{g}_{1} g_{3} \bar{g}_{1}\right)^{q-1} g_{2} \bar{g}_{1} g_{3},
\end{aligned}
$$

where $g_{2}=\bar{a} g_{1} a$ and $g_{3}=a g_{1} \bar{a}$. It follows that $G(J(2,2 q))$ is generated by the three conjugate elements $g_{1}, g_{2}$, and $g_{3}$.

Similarly, in the case $q<0$, we can show that $G(J(2,2 q))$ is generated by the three conjugate elements $g_{1}, g_{2}=\bar{b} g_{1} b, g_{3}=b g_{1} \bar{b}$. Here $b$ can be expressed as $\left(g_{3} \bar{g}_{1} g_{2} \bar{g}_{1}\right)^{q} g_{1}$.

Johnson's method for knot group epimorphs González-Acuña and Johnson showed independently the following.

Theorem $2.3[6 ; 12] \quad$ Let $G$ be a group finitely generated by the conjugates of $g \in G$. Then there is a knot with group $G(K)$ and meridian $\mu \in G(K)$, and a homomorphism $\varphi$ of $G(K)$ onto $G$ carrying $\mu$ onto $g$.

Johnson proved this result by presenting a process to construct such a knot $K$. Here we review this process.

By hypothesis, $G$ is finitely generated and normally generated by $g$. Thus we can choose finitely many generators of the form $g_{1}=g, g_{2}=w_{2} g \bar{w}_{2}, \ldots, g_{n}=w_{n} g \bar{w}_{n}$. Take a trivial link with $n$ components, and label the components by $g_{1}, g_{2}, \ldots, g_{n}$. For each $i \geq 2$, we connect the component $g_{i}$ to $g_{1}$ along a band which represents the word $w_{i}$; for example, if $w_{2}=g_{1} \bar{g}_{3}$, we connect the first circle and the second circle as in Figure 3. Let $K$ be the resulting knot. Then it is not too difficult to see that $G(K)$ satisfies the conclusion of Theorem 2.3.

For the twist knot group $G(J(2,2 q))$, we use the pseudo-meridian $g_{1}$ defined in Proposition 2.2. By the process of Theorem 2.3, we can construct a knot $J_{q}$ for which there exists an epimorphism $\varphi: G\left(J_{q}\right) \rightarrow G(J(2,2 q))$ that sends a meridian of $J_{q}$ to the pseudo-meridian $g_{1}$ of $J(2,2 q)$. In particular, $\varphi$ is non-meridional. 


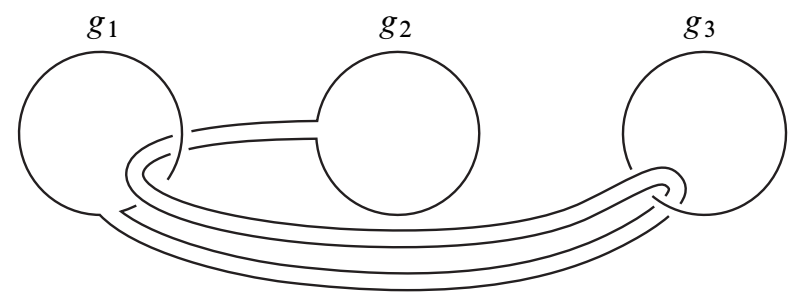

Figure 3: The connecting band for $w_{2}=g_{1} \bar{g}_{3}$.

\section{Twisted Alexander polynomial and nonexistence of merid- ional epimorphisms}

In this section we show that two of the examples described in Section 2 do not admit any meridional epimorphisms. For this purpose we use twisted Alexander polynomials, as discussed below.

\subsection{Obstructions to admitting a meridional epimorphism}

We recall the definition of the twisted Alexander polynomial, following Wada [22]. For this purpose, temporarily, we assume that a knot is oriented. For brevity, although any presentation of a knot group can be used in general, we will consider only the special case of a deficiency-one presentation

$$
G=\left\langle x_{1}, \ldots, x_{k} \mid r_{1}, \ldots, r_{k-1}\right\rangle
$$

of a knot group $G=G(K)$ in which each generator $x_{i}$ represents a positively oriented meridian. For instance, a Wirtinger presentation can be used. Suppose $\rho: G \rightarrow \operatorname{SL}(n, \mathbb{F})$ is a representation over a field $\mathbb{F}$. Let $\alpha: G \rightarrow \mathbb{Z}=\langle t\rangle$ be the surjection defined by $\alpha\left(x_{i}\right)=t$. The tensor representation $\rho \otimes \alpha$ defined by $(\rho \otimes \alpha)(g)=\alpha(g) \cdot \rho(g)$ gives rise to a ring homomorphism $\mathbb{Z}[G] \rightarrow M_{n}\left(\mathbb{F}\left[t, t^{-1}\right]\right)$. Let $F\left\langle x_{i}\right\rangle$ be the free group generated by the symbols $x_{1}, \ldots, x_{k}$, and let $\Phi$ be the composition

$$
\Phi: \mathbb{Z}\left[F\left\langle x_{i}\right\rangle\right] \stackrel{\text { proj. }}{\longrightarrow} \mathbb{Z}[G] \stackrel{\rho \otimes \alpha}{\longrightarrow} M_{n}\left(\mathbb{F}\left[t, t^{-1}\right]\right) .
$$

Viewing $r_{i}$ as an element of $F\left\langle x_{i}\right\rangle$, the Fox derivative $\partial r_{i} / \partial x_{j} \in \mathbb{Z}\left[F\left\langle x_{i}\right\rangle\right]$ is defined as in [5]. The matrix consisting of $(k-1) \times k$ blocks of size $n \times n$

$$
\Phi\left(\frac{\partial r_{i}}{\partial x_{j}}\right) \in M_{n}\left(\mathbb{F}\left[t, t^{-1}\right]\right), \quad 1 \leq i \leq k-1,1 \leq j \leq k
$$

is called a twisted Alexander matrix. It can be verified that det $\Phi\left(1-x_{j}\right) \neq 0$ for some $j$ (see [22, Lemma 2]). For such an index $j$, let $M_{j}$ be the matrix obtained from 


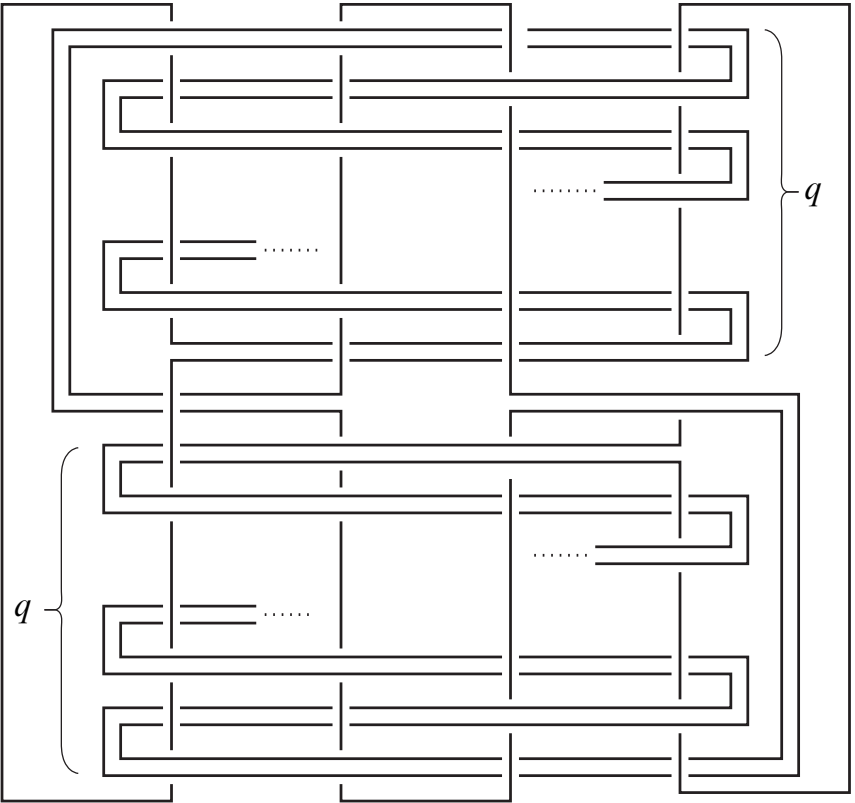

Figure 4: The knot $J_{q}(q>0)$.

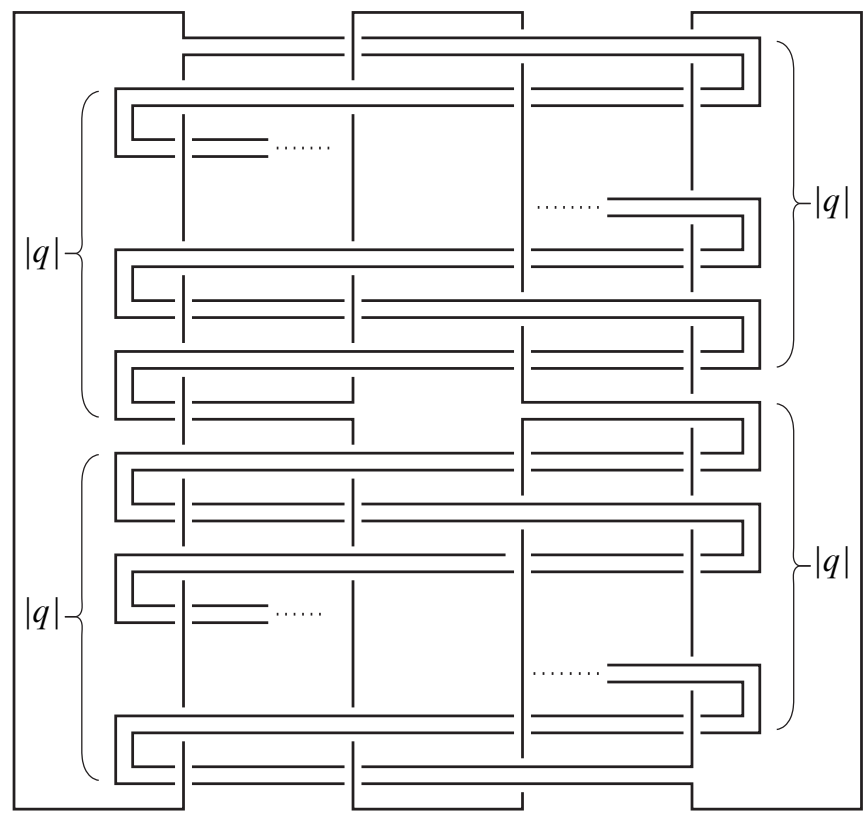

Figure 5: The knot $J_{q}(q<0)$. 
the twisted Alexander matrix by deleting the blocks on the $j^{\text {th }}$ column. Viewing $M_{j}$ as an $n(k-1) \times n(k-1)$ matrix over $\mathbb{F}\left[t, t^{-1}\right]$, we define the twisted Alexander polynomial for $(K, \rho)$ by $\Delta_{K, \rho}=\Delta_{K, \rho}^{N} / \Delta_{K, \rho}^{D}$, where

$$
\Delta_{K, \rho}^{N}=\operatorname{det}\left(M_{j}\right), \quad \Delta_{K, \rho}^{D}=\operatorname{det} \Phi\left(x_{j}-1\right) .
$$

We call $\Delta_{K, \rho}^{N}$ and $\Delta_{K, \rho}^{D}$ the numerator and denominator of the twisted Alexander polynomial. Note that $\Delta_{K, \rho}^{N}$ and $\Delta_{K, \rho}^{D}$ are Laurent polynomials in $\mathbb{F}\left[t, t^{-1}\right]$.

Under our assumption that the generators of the presentation for $G$ are positive meridians, both polynomials $\Delta_{K, \rho}^{N}$ and $\Delta_{K, \rho}^{D}$ are well-defined invariants of $(K, \rho)$, up to multiplication by units in $\mathbb{F}\left[t, t^{-1}\right]$. This is a consequence of the following two facts: (i) the fraction $\Delta_{K, \rho}$ is well-defined up to units for any choice of a presentation of $G$ (see eg [22]), and (ii) $\Delta_{K, \rho}^{D}=\operatorname{det} \Phi\left(x_{j}-1\right)$ is determined, up to units, by the conjugacy class of the generator $x_{j}$.

Remark 3.1 By the same argument, the following more general statement is true: for a finitely presentable group $G$ and a conjugacy class $c$, the numerator and denominator of the twisted Alexander polynomial of $G$ are invariants of $(G, c)$, provided that we use a presentation whose generators are in the conjugacy class $c$.

Remark 3.2 For an unoriented knot $K, \Delta_{K, \rho}^{N}$ and $\Delta_{K, \rho}^{D}$ are well-defined up to the substitution $t \rightarrow t^{-1}$ (and up to multiplication by a unit). For our purpose, this does not cause any problem.

The following theorem of Kitano, Suzuki and Wada is the key ingredient we use to detect the non-existence of a meridional epimorphism.

Theorem 3.3 [16] Suppose there is a meridional epimorphism $G(K) \rightarrow G\left(K^{\prime}\right)$. Then for any representation $\rho^{\prime}: G\left(K^{\prime}\right) \rightarrow \operatorname{SL}(n, \mathbb{F})$ over a field $\mathbb{F}$, there is a representation $\rho: G(K) \rightarrow \operatorname{SL}(n, \mathbb{F})$ such that for some $\epsilon \in\{1,-1\}, \Delta_{K^{\prime}, \rho^{\prime}}^{N}(t)$ divides $\Delta_{K, \rho}^{N}\left(t^{\epsilon}\right)$ in $\mathbb{F}\left[t, t^{-1}\right]$ and $\Delta_{K^{\prime}, \rho^{\prime}}^{D}(t)$ is equal to $\Delta_{K, \rho}^{D}\left(t^{\epsilon}\right)$ up to units.

Remark 3.4 (1) The representation $\rho$ in Theorem 3.3 is the composition of the given meridional epimorphism and $\rho^{\prime}$. Since our aim is to detect the nonexistence of a meridional epimorphism, we do not know what $\rho$ would be. Consequently, to use Theorem 3.3. we need to investigate all representations $\rho: G(K) \rightarrow \operatorname{SL}(n, \mathbb{F})$ such that $\operatorname{Im} \rho=\operatorname{Im} \rho^{\prime}$ and $\rho$ (meridian of $K$ ) is conjugate to $\rho$ (meridian of $K^{\prime}$ ) in $\operatorname{Im} \rho^{\prime}$.

(2) Under the weaker assumption that there is an epimorphism $G(K) \rightarrow G\left(K^{\prime}\right)$, which might be non-meridional, we have the weaker conclusion that $\Delta_{K^{\prime}, \rho^{\prime}}(t)$ divides $\Delta_{K, \rho}\left(t^{\epsilon}\right)$. See [16]. 


\subsection{Computation for the first example on the trefoil}

In this subsection we show that there is no meridional epimorphism of the knot group $G\left(K_{T}\right)$ onto the trefoil group $G(T)$, where $K_{T}$ is the knot presented in Figure 1 .

We will use representations over $\operatorname{SL}\left(2, \mathbb{F}_{5}\right)$. In particular, consider the representation

$$
\rho^{\prime}: G(T)=\left\langle y_{1}, y_{2} \mid y_{1} y_{2} y_{1}=y_{2} y_{1} y_{2}\right\rangle \longrightarrow \operatorname{SL}\left(2, \mathbb{F}_{5}\right)
$$

defined by

$$
\rho^{\prime}\left(y_{1}\right)=\left[\begin{array}{ll}
0 & 4 \\
1 & 3
\end{array}\right], \quad \rho^{\prime}\left(y_{2}\right)=\left[\begin{array}{ll}
0 & 1 \\
4 & 3
\end{array}\right] .
$$

It is straightforward to verify that the relator is sent to the identity.

A computer-aided computation shows that

$$
\Delta_{E, \rho^{\prime}}^{N}=t^{4}+2 t^{3}+2 t^{2}+2 t+1, \quad \Delta_{E, \rho^{\prime}}^{D}=t^{2}+2 t+1 .
$$

Note that both $\Delta_{T, \rho^{\prime}}^{N}$ and $\Delta_{T, \rho^{\prime}}^{D}$ are symmetric.

In order to invoke Theorem 3.3, we compute the twisted Alexander polynomials of $K_{T}$. We are again aided by computer programs written by the authors, which enumerates all the representations $\rho: G\left(K_{T}\right) \rightarrow \operatorname{SL}\left(2, \mathbb{F}_{5}\right)$ (up to conjugation) and then computes the associated twisted Alexander polynomials. By this we obtain that there are exactly eight distinct twisted Alexander polynomials of $G\left(K_{T}\right)$ over $\operatorname{SL}\left(2, \mathbb{F}_{5}\right)$. These polynomials are listed in the appendix in Table 1.

From Table 1, it is straightforward to verify that for any representation $\rho: G\left(K_{T}\right) \rightarrow$ $\operatorname{SL}\left(2, \mathbb{F}_{5}\right)$,

$$
\Delta_{K_{T}, \rho}^{D} \neq \Delta_{T, \rho^{\prime}}^{D} \quad \text { or } \quad \Delta_{K_{T}, \rho}^{N} \text { does not divide } \Delta_{T, \rho^{\prime}}^{N} .
$$

By symmetry of the polynomials, the conclusion holds for $\left(\Delta_{T, \rho^{\prime}}^{N}\left(t^{-1}\right), \Delta_{T, \rho^{\prime}}^{D}\left(t^{-1}\right)\right)$ as well. By Theorem 3.3, it follows that there is no meridional epimorphism of $G\left(K_{T}\right)$ onto $G(T)$.

\subsection{Computation for $J_{-1}$ and the figure-eight knot}

In this subsection we show that there is no meridional epimorphism of the knot group $G\left(J_{-1}\right)$ onto the figure-eight group $G(E)$, where $J_{-1}$ is the knot described in Section 2.2.

For this case, we use representations over $\operatorname{SL}\left(2, \mathbb{F}_{7}\right)$. Let

$$
\rho^{\prime}: G(E)=\left\langle y_{1}, y_{2} \mid \bar{y}_{1} \bar{y}_{2} y_{1} y_{2} \bar{y}_{1} y_{2} y_{1} \bar{y}_{2} \bar{y}_{1} y_{2}\right\rangle \longrightarrow \operatorname{SL}\left(2, \mathbb{F}_{7}\right)
$$


be the representation defined by

$$
\rho^{\prime}\left(y_{1}\right)=\left[\begin{array}{ll}
0 & 4 \\
5 & 2
\end{array}\right], \quad \rho^{\prime}\left(y_{2}\right)=\left[\begin{array}{ll}
1 & 0 \\
3 & 1
\end{array}\right] .
$$

The relator of $G(E)$ is sent to the identity, and we have

$$
\Delta_{E, \rho^{\prime}}^{N}=t^{4}+t^{3}+3 t^{2}+t+1, \quad \Delta_{E, \rho^{\prime}}^{D}=t^{2}+5 t+1 .
$$

By computation aided by a computer, we obtain that there are exactly 139 representations up to conjugacy, and 58 distinct twisted Alexander polynomials of $G\left(J_{-1}\right)$ over $\operatorname{SL}\left(2, \mathbb{F}_{7}\right)$. We discuss this in the appendix, and a table of the polynomials can be found in the online supplement [3]. From this table it is verified that for any representation $\rho: G\left(J_{-1}\right) \rightarrow \operatorname{SL}\left(2, \mathbb{F}_{7}\right)$, either

$$
\Delta_{J_{-1}, \rho}^{D} \neq \Delta_{E, \rho^{\prime}}^{D} \quad \text { or } \quad \Delta_{J_{-1}, \rho}^{N} \text { does not divide } \Delta_{E, \rho^{\prime}}^{N} .
$$

Since both polynomials $\Delta_{E, \rho^{\prime}}^{D}(t)$ and $\Delta_{E, \rho^{\prime}}^{N}(t)$ are symmetric, the conclusion holds also for $\left(\Delta_{E, \rho^{\prime}}^{N}\left(t^{-1}\right), \Delta_{E, \rho^{\prime}}^{D}\left(t^{-1}\right)\right)$. By Theorem 3.3, it follows that there is no meridional epimorphism of $G\left(J_{-1}\right)$ onto $G(E)$.

Remark 3.5 The most time-consuming step of the computation is to find all the representations of the given knot group. Our implementation performs a brute-force search; since its running time is exponential in the number of the generators, it would be interesting if a more clever algorithm were available. For $G\left(K_{T}\right)$, there is a presentation with 3 generators, and all the $37 \mathrm{SL}\left(2, \mathbb{F}_{5}\right)$ representations (up to conjugacy) are found within a few seconds. For $G\left(J_{-1}\right)$, we use a simplified presentation with 5 generators, and all the $139 \operatorname{SL}\left(2, \mathbb{F}_{7}\right)$ representations (up to conjugacy) are found within 2 minutes. For $G\left(J_{q}\right)$ for $q \geq 1$ or $q<-1$, we could not derive any conclusion within reasonable running time; computation for two weeks on a computer with a $3 \mathrm{GHz}$ Intel i7 processor was not enough.

\section{Satellite construction and knot group epimorphisms}

In this section we present a method to obtain infinitely many pairs of knots $\left(K, K^{\prime}\right)$ for which $G\left(K^{\prime}\right)$ is a non-meridional homomorph of $G(K)$ but there is no meridional epimorphism $G(K) \rightarrow G\left(K^{\prime}\right)$. We will start with a given "seed" pair $\left(K, K^{\prime}\right)$ of knots with the desired property, and then apply certain satellite constructions to produce infinitely many such examples. 


\subsection{Satellite construction and knot group epimorphisms}

We begin by recalling the standard satellite construction. Let $K$ be a knot in $S^{3}$ and $\alpha$ an unknotted oriented embedded circle in $S^{3}$ disjoint from $K$. Glue the exterior $E_{\alpha}$ of $\alpha \subset S^{3}$ and the exterior $E_{J}$ of another knot $J$ in $S^{3}$ along an orientation-reversing diffeomorphism between the boundary tori, which identifies a zero-linking longitude and positively oriented meridian of $\alpha$ with a positively oriented meridian and zero-linking longitude of $J$, respectively. There is a diffeomorphism of the resulting 3-manifold onto $S^{3}$, and the image of $K$ under the diffeomorphism is a new knot, which we denote by $K(\alpha, J)$. In traditional terminology, $J$ is the companion and $K$ viewed as a knot in the solid torus $E_{\alpha}$ is the pattern. As illustrated in Figure $6, K(\alpha, J)$ is the knot obtained by "tying" $J$ into $K$ along a 2-disk bounded by $\alpha$.

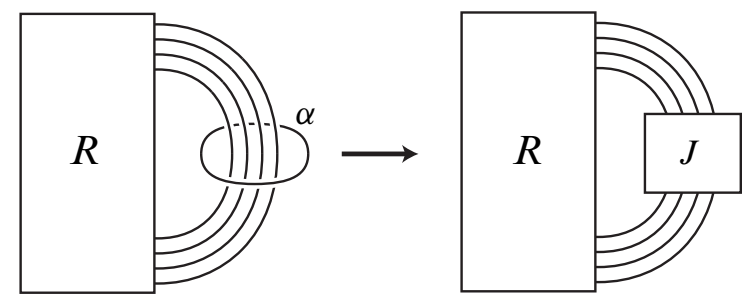

Figure 6: A satellite construction.

Note that $E_{K \sqcup \alpha}$ is a subspace of $E_{K(\alpha, J)}$ and a subspace of $E_{K}$. Let $i_{*}$ and $j_{*}$ be the inclusion-induced homomorphisms of $G(K \sqcup \alpha)$ into $G(K(\alpha, J))$ and $G(K)$, respectively. Then we have the following folklore:

Lemma 4.1 There exists a meridional epimorphism $q: G(K(\alpha, J)) \rightarrow G(K)$ satisfying $i_{*}=q \circ j_{*}$, ie so that the following diagram commutes:

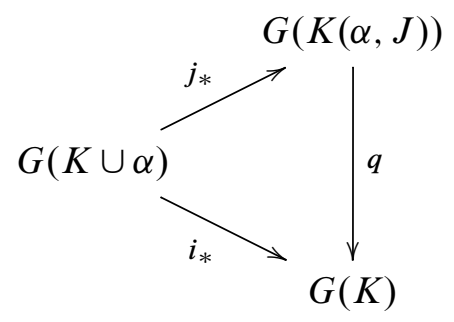

For the reader's convenience, we give a proof.

Proof There is a degree-one map $E_{J}$ to the trivial knot exterior $S^{1} \times D^{2}$ which extends a diffeomorphism on the boundary sending the meridian and zero-linking 
longitude of $J$ to those of the unknot. Gluing it with the identity map on $E_{K \cup \alpha}$, one obtains a degree-one map $E_{K(\alpha, J)} \rightarrow E_{K}$ which induces the desired $q$.

We recall some standard definitions. The derived subgroups of a group $G$ are defined inductively by $G^{(0)}=G$ and $G^{(n+1)}=\left[G^{(n)}, G^{(n)}\right]$ for $n \geq 0$, where $[A, B]$ denotes the subgroup generated by commutators $\left\{a b a^{-1} b^{-1} \mid a \in A, b \in B\right\}$. For the first infinite ordinal $\omega$, the transfinite derived subgroup $G^{(\omega)}$ is defined by $G^{(\omega)}=\bigcap_{n<\infty} G^{(n)}$. A group $G$ is residually solvable if $G^{(\omega)}=\{e\}$.

Remark 4.2 (1) The following well-known examples will be useful for our purposes: if $K$ is a fibered knot (or link), then $G(K)$ is residually solvable, for the commutator subgroup $G(K)^{(1)}=[G(K), G(K)]$ is the fundamental group of a surface with nonempty boundary, and thus a free group. It is known that a free group is residually solvable.

(2) Not all knot groups are residually solvable. For example, the group of a nontrivial knot $K$ with Alexander polynomial 1 is not residually solvable.

The key technical ingredient we use is the following.

Theorem 4.3 Suppose $T$ is a knot with residually solvable group $G(T)$. Suppose $J$ has Alexander polynomial 1. Then for any homomorphism $f: G(K(\alpha, J)) \rightarrow G(T)$, there is an induced homomorphism $f^{\prime}: G(K) \rightarrow G(T)$ that makes the following diagram commute:

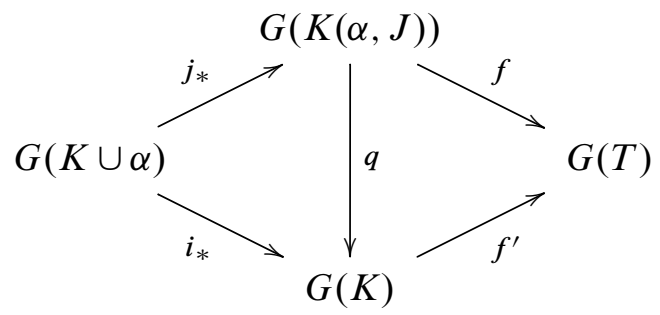

In addition, the following hold:

(1) If $f$ is an epimorphism, then $f^{\prime}$ is an epimorphism.

(2) If $f$ is meridional, then $f^{\prime}$ is meridional.

The following corollary is an immediate consequence.

Corollary 4.4 If $T$ and $J$ are as in Theorem 4.3 and there is no meridional epimorphism of $G(K)$ onto $G(T)$, then there is no meridional epimorphism of $G(K(\alpha, J))$ onto $G(T)$. 
Proof of Theorem 4.3 Consider the composition

$$
g: G(J) \longrightarrow G(K(\alpha, J)) \longrightarrow G(T) .
$$

Since $J$ has Alexander polynomial 1, the commutator subgroup $[G(J), G(J)]$ is perfect, ie $G(J)^{(1)}=G(J)^{(2)}=\cdots=G(J)^{(\omega)}$. Since $g\left(G(J)^{(\omega)}\right) \subset G(T)^{(\omega)}$ and $G(T)$ is residually solvable, it follows that $G(J)^{(1)} \subset \operatorname{Ker} g$. Therefore $g$ factors through $G(J) /[G(J), G(J)]=\mathbb{Z}$.

From the construction of $K(\alpha, J)$, one sees that $G(K(\alpha, J))$ is the amalgamated product of $G(K \cup \alpha)$ and $G(J)$ over $\pi_{1}\left(S^{1} \times S^{1}\right)=\mathbb{Z}^{2}$. The homomorphism

$$
G(K(\alpha, J))=G(K \cup \alpha) \amalg_{\mathbb{Z}^{2}} G(J) \longrightarrow G(K \cup \alpha) \amalg_{\mathbb{Z}^{2}} \mathbb{Z}=G(K)
$$

induced by the abelianization $G(J) \rightarrow \mathbb{Z}$ is our $q$ in Lemma 4.1. From the observation in the previous paragraph, it follows that $f: G(K(\alpha, J)) \rightarrow G(T)$ induces a homomorphism from $G(K)$ to $G(T)$. This proves the first conclusion.

From the commutative diagram the remaining conclusions follow immediately.

\subsection{Infinitely many examples}

Theorem 4.5 Let $T$ be the trefoil knot or the figure-eight knot. Then there are infinitely many prime knots $K_{1}, K_{2}, \ldots$ satisfying the following:

(1) For each $n$ there is an epimorphism $G\left(K_{n}\right) \rightarrow G(T)$ but there is no meridional epimorphism $G\left(K_{n}\right) \rightarrow G(T)$.

(2) $K_{n}$ and $K_{m}$ are not equivalent for any $n \neq m$.

Remark 4.6 Due to Silver and Whitten [20], for prime knots there exists a meridional epimorphism if and only if there exists an epimorphism preserving peripheral subgroups. Therefore, for our $K_{n}$ in Theorem 4.5, there is no epimorphism $G\left(K_{n}\right) \rightarrow G(T)$ sending peripheral subgroups into peripheral subgroups.

Proof Recall that we have constructed a prime knot $K$ which admits an epimorphism $G(K) \rightarrow G(T)$ but does not admit any meridional epimorphism $G(K) \rightarrow G(T)$; when $T$ is the trefoil, $K=K_{T}$ in Figure 1, and when $T$ is the figure-eight, $K=J_{-1}$ in Figure 5.

Choose an embedded circle $\alpha$ in $S^{3}-K$ satisfying the following: $\alpha$ is unknotted in $S^{3}$, $\alpha$ does not bound a 2-disk in $E_{K}$, and $K \cup \alpha$ is a prime link. For example, we may use $\alpha$ as shown in Figures 7 and 8. Choose a hyperbolic nontrivial knot $P$ with Alexander polynomial 1; for example, the Conway knot 11n_34 or the Kinoshita-Terasaka knot 


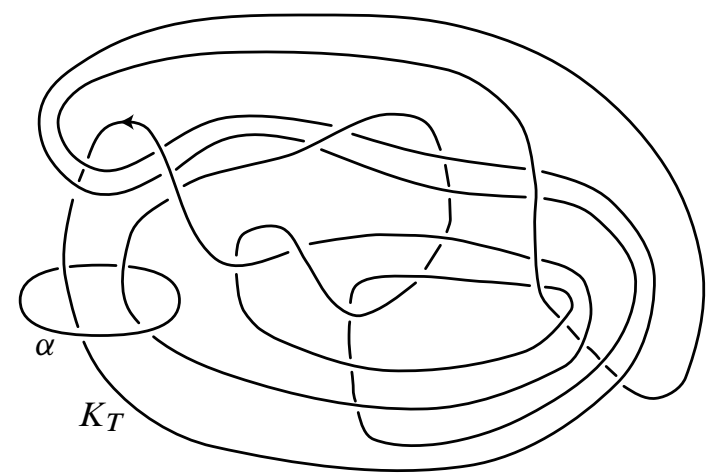

Figure 7: A curve $\alpha$ for a satellite construction on $K_{T}$. The link $K_{T} \cup \alpha$ is hyperbolic with volume 23.2123, according to SnapPy [4].

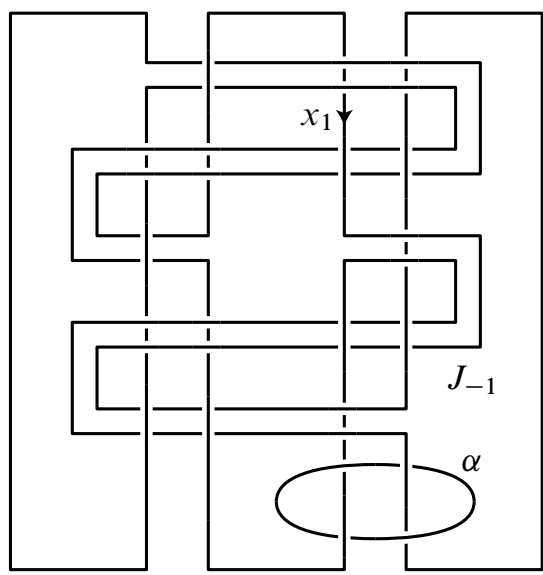

Figure 8: A curve $\alpha$ for a satellite construction on $J_{-1}$. The link $J_{-1} \cup \alpha$ is hyperbolic with volume 26.2914, according to SnapPy [4].

11n_42 in KnotInfo [2] can be used as $P$. Let $P_{n}$ be the connected sum of $n$ copies of $P$. Let $K_{n}=K\left(\alpha, P_{n}\right)$.

Note that $G(T)$ is residually solvable since $T$ is fibered. Since the seed knot $K$ satisfies the conclusion (1), our $K_{n}$ satisfies (1) by Lemma 4.1 and Theorem 4.3.

To show that the $K_{n}$ have distinct knot types, we consider the JSJ decomposition of the exterior. Indeed, in most cases satellite constructions with distinct companion give distinct JSJ decompositions. In our case this can be seen as follows. The exterior of $E_{K_{n}}$ is the union of $E_{K \cup \alpha}$ and $E_{P_{n}}$. Since $P_{n}$ is a nontrivial knot and $\alpha$ does not bound a 2-disk in $E_{K}$, the boundary of $E_{P_{n}}$ is an incompressible torus in $E_{K_{n}}$. Since $P$ is hyperbolic, the JSJ tori of $E_{K_{n}}$ are exactly the union of $\partial E_{P_{n}}$ and the JSJ 
tori of $E_{K \cup \alpha}$ and $E_{P_{n}}$. In addition, since $P_{n}$ is the connected sum of $n$ copies of the hyperbolic knot $P$, the number of the JSJ tori of $E_{K_{n}}$ is monotonically increasing. It follows that $K_{n}$ and $K_{m}$ have non-homeomorphic exteriors if $n \neq m$.

For the primality of the $K_{n}$, one may again look at the JSJ decomposition: since $K \cup \alpha$ is prime, the root piece in the JSJ decomposition of $E_{K_{n}}$ is not a composition space and therefore $K_{n}$ is not a composite knot.

\section{Appendix: Tables of twisted Alexander polynomials}

\section{A.1 Twisted Alexander polynomials of $K_{T}$ over $\operatorname{SL}\left(2, \mathbb{F}_{5}\right)$}

In this subsection we present all the twisted Alexander polynomials, associated to representations over $\operatorname{SL}\left(2, \mathbb{F}_{5}\right)$, of the knot $K_{T}$ shown in Figure 1.

We use a simpler presentation of $G\left(K_{T}\right)$ to find all the representations. A straightforward simplification of the presentation of $G\left(K_{T}\right)$ used in Section 2.1 shows that $x_{1}, x_{6}$, and $x_{17}$ generate $G\left(K_{T}\right)$. In fact, these three generators and the following relators form a presentation of $G\left(K_{T}\right)$ :

$$
\begin{aligned}
& x_{1} x_{17} \bar{x}_{1} x_{6}\left(x_{17} \bar{x}_{6} x_{1} \bar{x}_{17} \bar{x}_{1}\right)^{3} x_{17} \bar{x}_{1} \bar{x}_{6}\left(x_{1} \bar{x}_{17} x_{1} x_{17} \bar{x}_{1} x_{6}\right)^{2} \\
& \cdot\left(\bar{x}_{17} x_{1} x_{17} \bar{x}_{1} x_{6}\right)^{2} \bar{x}_{17}\left(\bar{x}_{6} x_{1} \bar{x}_{17} \bar{x}_{1}\right)^{2} x_{6}, \\
& x_{1}\left(\bar{x}_{17} x_{1} x_{17} \bar{x}_{1} x_{6}\right)^{3} \bar{x}_{17}\left(\bar{x}_{6} x_{1} \bar{x}_{17} \bar{x}_{1} x_{17}\right)^{4} \\
& \cdot \bar{x}_{1} \bar{x}_{6} x_{1}\left(\bar{x}_{17} x_{1} x_{17} \bar{x}_{1} x_{6}\right)^{4}\left(x_{17} \bar{x}_{6} x_{1} \bar{x}_{17} \bar{x}_{1}\right)^{3} x_{17} .
\end{aligned}
$$

We use computer programs to find all the representations over $\operatorname{SL}\left(2, \mathbb{F}_{5}\right)$ and to compute the associated twisted Alexander polynomials. It turns out that there are exactly 19 representations modulo conjugacy, which give 8 distinct twisted Alexander polynomials. We list the 8 polynomials in Table 1. For each twisted Alexander polynomial in Table 1, we describe a representation $\rho: G\left(K_{T}\right) \rightarrow \operatorname{SL}\left(2, \mathbb{F}_{5}\right)$ (which is not unique in general) which gives the polynomial by presenting the matrices $\rho\left(x_{1}\right), \rho\left(x_{6}\right)$, and $\rho\left(x_{17}\right)$.

\section{A.2 Twisted Alexander polynomials of $J_{-1}$ over $\operatorname{SL}\left(2, \mathbb{F}_{7}\right)$}

In this subsection we discuss the twisted Alexander polynomials, over $\operatorname{SL}\left(2, \mathbb{F}_{7}\right)$, of the knot $J_{-1}$ given in Figure 5. A full table of these polynomials is given in an online supplement [3]. We start with the standard Wirtinger representation of $J_{-1}$; the generators are labeled by $x_{1}, x_{2}, \ldots, x_{32}$, starting from the arc with an arrow in Figure 5. By simplifying the Wirtinger presentation, we obtain a deficiency-one presentation 


\begin{tabular}{lcccc}
$\rho\left(x_{1}\right)$ & $\rho\left(x_{6}\right)$ & $\rho\left(x_{17}\right)$ & $\Delta_{K_{T}, \rho}^{N}$ & $\Delta_{K_{T}, \rho}^{D}$ \\
\hline$\left[\begin{array}{ll}1 & 0 \\
0 & 1\end{array}\right]$ & {$\left[\begin{array}{ll}1 & 0 \\
0 & 1\end{array}\right]$} & {$\left[\begin{array}{ll}1 & 0 \\
0 & 1\end{array}\right]$} & $t^{8}+t^{7}+4 t^{5}+4 t^{4}+4 t^{3}+t+1$ & $t^{2}+3 t+1$ \\
{$\left[\begin{array}{ll}2 & 4 \\
4 & 1\end{array}\right]$} & {$\left[\begin{array}{ll}2 & 4 \\
4 & 1\end{array}\right]$} & {$\left[\begin{array}{ll}2 & 4 \\
4 & 1\end{array}\right]$} & $t^{8}+4 t^{7}+t^{5}+4 t^{4}+t^{3}+4 t+1$ & $t^{2}+2 t+1$ \\
{$\left[\begin{array}{ll}0 & 2 \\
2 & 4\end{array}\right]$} & {$\left[\begin{array}{ll}0 & 2 \\
2 & 4\end{array}\right]$} & {$\left[\begin{array}{ll}0 & 2 \\
2 & 4\end{array}\right]$} & $t^{8}+2 t^{7}+t^{6}+2 t^{5}+4 t^{4}+2 t^{3}+t^{2}+2 t+1$ & $t^{2}+t+1$ \\
{$\left[\begin{array}{ll}0 & 2 \\
2 & 4\end{array}\right]$} & {$\left[\begin{array}{ll}0 & 4 \\
1 & 4\end{array}\right]$} & {$\left[\begin{array}{ll}1 & 3 \\
4 & 3\end{array}\right]$} & $t^{8}+2 t^{7}+t^{5}+3 t^{4}+t^{3}+2 t+1$ & $t^{2}+t+1$ \\
{$\left[\begin{array}{ll}1 & 1 \\
4 & 0\end{array}\right]$} & {$\left[\begin{array}{ll}1 & 1 \\
4 & 0\end{array}\right]$} & {$\left[\begin{array}{ll}1 & 1 \\
4 & 0\end{array}\right]$} & $t^{8}+3 t^{7}+t^{6}+3 t^{5}+4 t^{4}+3 t^{3}+t^{2}+3 t+1$ & $t^{2}+4 t+1$ \\
{$\left[\begin{array}{ll}1 & 1 \\
4 & 0\end{array}\right]$} & {$\left[\begin{array}{ll}2 & 2 \\
1 & 4\end{array}\right]$} & {$\left[\begin{array}{ll}3 & 4 \\
2 & 3\end{array}\right]$} & $t^{8}+3 t^{7}+4 t^{5}+3 t^{4}+4 t^{3}+3 t+1$ & $t^{2}+4 t+1$ \\
{$\left[\begin{array}{ll}3 & 2 \\
0 & 2\end{array}\right]$} & {$\left[\begin{array}{ll}3 & 2 \\
0 & 2\end{array}\right]$} & {$\left[\begin{array}{ll}3 & 2 \\
0 & 2\end{array}\right]$} & $t^{8}+3 t^{6}+3 t^{4}+3 t^{2}+1$ & $t^{2}+1$ \\
{$\left[\begin{array}{ll}3 & 2 \\
0 & 2\end{array}\right]$} & {$\left[\begin{array}{ll}0 & 3 \\
3 & 0\end{array}\right]$} & {$\left[\begin{array}{ll}0 & 3 \\
3 & 0\end{array}\right]$} & $t^{8}+4 t^{6}+t^{4}+4 t^{2}+1$ & $t^{2}+1$
\end{tabular}

Table 1: The twisted Alexander polynomials of the knot $K_{T}$ in Figure 1 over $\operatorname{SL}\left(2, \mathbb{F}_{5}\right)$.

of $G\left(J_{-1}\right)$ whose generators are the Wirtinger generators $x_{2}, x_{10}, x_{19}, x_{22}, x_{30}$, and whose relators are the following four words:

$x_{19} \bar{x}_{30} \bar{x}_{10} x_{30} \bar{x}_{19} x_{10} \bar{x}_{2} x_{30} x_{2} \bar{x}_{30} x_{2} \bar{x}_{10} x_{19} \bar{x}_{30} x_{10} x_{30} \bar{x}_{19} x_{10} \bar{x}_{2} \bar{x}_{30} x_{2} \bar{x}_{10}$, $x_{30} x_{19} \bar{x}_{30} \bar{x}_{10} x_{30} \bar{x}_{19} x_{22}\left(x_{30} \bar{x}_{19}\right)^{2}\left(\bar{x}_{30} x_{19}\right)^{2}$

$\cdot \bar{x}_{30} \bar{x}_{22} x_{19} \bar{x}_{30} x_{10} x_{30} \bar{x}_{19} \bar{x}_{30} x_{2} \bar{x}_{10} x_{22} x_{10} \bar{x}_{2}$,

$\bar{x}_{30} x_{2} \bar{x}_{10} x_{19} \bar{x}_{30} x_{10} x_{30} \bar{x}_{19} \bar{x}_{30} x_{2} \bar{x}_{10} x_{22} x_{10} x_{2} \bar{x}_{10} \bar{x}_{22}$

$\cdot\left(x_{10} \bar{x}_{2} x_{30} x_{19} \bar{x}_{30} \bar{x}_{10} x_{30} \bar{x}_{19}\right)^{2} x_{10} \bar{x}_{2} x_{30} \bar{x}_{10}$, $x_{19} \bar{x}_{30} \bar{x}_{22} x_{19} \bar{x}_{30} x_{10} x_{2} \bar{x}_{10} \bar{x}_{22} x_{10} \bar{x}_{2} x_{30} x_{19} \bar{x}_{30} \bar{x}_{10}\left(x_{30} \bar{x}_{19} x_{22}\right)^{2} x_{19} \bar{x}_{30} \bar{x}_{22} x_{19} \bar{x}_{30}$ $\cdot x_{10} x_{30} \bar{x}_{19} \bar{x}_{30} x_{2} \bar{x}_{10} x_{22} x_{10} \bar{x}_{2} \bar{x}_{10} x_{30} \bar{x}_{19} x_{22}\left(x_{30} \bar{x}_{19}\right)^{2} \bar{x}_{30}$.

Again, we use computer programs which enumerate all the representations of this presentation over $\operatorname{SL}\left(2, \mathbb{F}_{7}\right)$ and compute the associated twisted Alexander polynomials. There are in total 139 representations up to conjugation, from which 58 distinct twisted Alexander polynomials are obtained. The polynomials are listed in the online supplement [3], along with a description for each polynomial of a representation $\rho$ (which is not unique in general) which gives it.

\section{References}

[1] I Agol, Y Liu, Presentation length and Simon's conjecture, J. Amer. Math. Soc. 25 (2012) 151-187 MR2833481

[2] J C Cha, C Livingston, KnotInfo: table of knot invariants, electronic resource (2015) Available at http://www.indiana.edu/ knotinfo 
[3] J C Cha, M Suzuki, Table of twisted Alexander polynomials of $J_{-1}$ over $\operatorname{SL}\left(2, \mathbb{F}_{7}\right)$ (2016) online supplement Available at http://tbd

[4] M Culler, NM Dunfield, JR Weeks, SnapPy, a computer program for studying the geometry and topology of 3-manifolds (2014) Available at http:// snappy.computop.org/

[5] R H Fox, Free differential calculus, I: Derivation in the free group ring, Ann. of Math. 57 (1953) 547-560 MR0053938

[6] F González-Acuña, Homomorphs of knot groups, Ann. of Math. 102 (1975) 373-377 MR0379671

[7] F González-Acuña, A Ramírez, Two-bridge knots with property Q, Q. J. Math. 52 (2001) 447-454 MR1874490

[8] F González-Acuña, A Ramírez, Epimorphisms of knot groups onto free products, Topology 42 (2003) 1205-1227 MR1981354

[9] K Horie, T Kitano, M Matsumoto, M Suzuki, A partial order on the set of prime knots with up to 11 crossings, J. Knot Theory Ramifications 20 (2011) 275-303 MR2782704

[10] J Hoste, P D Shanahan, Trace fields of twist knots, J. Knot Theory Ramifications 10 (2001) 625-639 MR1831680

[11] J Hoste, P D Shanahan, Epimorphisms and boundary slopes of 2-bridge knots, Algebr. Geom. Topol. 10 (2010) 1221-1244 MR2653061

[12] D Johnson, Homomorphs of knot groups, Proc. Amer. Math. Soc. 78 (1980) 135-138 MR548101

[13] D Johnson, C Livingston, Peripherally specified homomorphs of knot groups, Trans. Amer. Math. Soc. 311 (1989) 135-146 MR942427

[14] T Kitano, M Suzuki, A partial order in the knot table, Experiment. Math. 14 (2005) 385-390 MR2193401

[15] T Kitano, M Suzuki, A partial order in the knot table, II, Acta Math. Sin. (Engl. Ser.) 24 (2008) 1801-1816 MR2453061

[16] T Kitano, M Suzuki, M Wada, Twisted Alexander polynomials and surjectivity of a group homomorphism, Alg. Geom. Topol. 5 (2005) 1315-1324 MR2171811 Correction in Alg. Geom. Topol. 11 (2011) 2937-2939

[17] D Lee, M Sakuma, Epimorphisms between 2-bridge link groups: homotopically trivial simple loops on 2-bridge spheres, Proc. Lond. Math. Soc. 104 (2012) 359-386 MR2880244

[18] T Ohtsuki, R Riley, M Sakuma, Epimorphisms between 2-bridge link groups, from "The Zieschang Gedenkschrift" (M Boileau, M Scharlemann, R Weidmann, editors), Geom. Topol. Monogr. 14 (2008) 417-450 MR2484712 
[19] R Riley, Parabolic representations of knot groups, I, Proc. London Math. Soc. 24 (1972) 217-242 MR0300267

[20] D S Silver, W Whitten, Knot group epimorphisms, J. Knot Theory Ramifications 15 (2006) 153-166 MR2207903

[21] D S Silver, W Whitten, Knot group epimorphisms, II, preprint (2008) arXiv: 0806.3223

[22] M Wada, Twisted Alexander polynomial for finitely presentable groups, Topology 33 (1994) 241-256 MR1273784

JCC: Department of Mathematics, Pohang University of Science and Technology Gyeongbuk, Pohang 790-784, South Korea

JCC: School of Mathematics, Korea Institute for Advanced Study

Seoul 130-722, South Korea

MS: Department of Frontier Media Science, Meiji University

4-21-1 Nakano, Tokyo 164-8525, Japan

jccha@postech.ac.kr, macky@fms.meiji.ac.jp

Received: 16 May 2015 Revised: 1 June 2015 
